\title{
Utilizing a Bioscaffold for Socket Preservation: A Case Report
}

\author{
Adhikari $\mathrm{K}^{1^{*}}$, Tandukar $\mathrm{A}^{2}$, Kumar B, and Khanal B ${ }^{3}$ \\ ${ }^{1}$ Lecturer, Department of Periodontology and Oral Implantology, CODS, UCMS, Bhairahawa, Nepal \\ ${ }^{2}$ Inspector, Nepal police Hospital, Kathmandu, Nepal \\ ${ }^{3}$ Lecturer, Department of Prosthodontics, CODS, UCMS, Bhairahawa, Nepal
}

\begin{abstract}
Socket preservation technique preserves the soft and the hard tissues after tooth extraction; hence minimizing the need for any augmentation procedures in the future. Platelet Rich Fibrin (PRF) enhances the osteogenic differentiation and the healing process so can be utilised for socket preservation. This is a case report of utilizing PRF in combination with hydroxyapatite crystals in an extracted socket, to preserve the future implant site. The tooth was extracted atraumatically and the socket was thoroughly debrided. Venous blood was collected from the patient's antecubital fossa and PRF was prepared immediately by centrifuging the collected blood at $3000 \mathrm{rpm}$ for 10 minutes. A mixture of hydroxyapatite and PRF was placed in the socket; covered by a PRF membrane and sutured with 3-0 silk suture. The radiographic and clinical evaluation demonstrated satisfactory regeneration of bone and soft tissue. Socket preservation technique using PRF and hydroxyapatite is an effective method for achieving sound bone and tissue for implant placement.
\end{abstract}

Key words: extraction, hydroxyapatite, implant, PRF, socket preservation

\section{Introduction}

A lveolar bone is lost following tooth extraction, periodontal diseases or any traumatic injury. The healing of which takes place generally by formation of connective tissue instead of bone, Inadequate horizontal and vertical bone may result in major clinical problems $^{1}$ and need to be corrected before placing the implant. A preexisting periodontal or endodontic infection or trauma from extraction often destroys labial plate and causes immediate loss of width and height of bone, which may exceed $50 \%$ of optimum bone volume ${ }^{2}$. To overcome the reduced bone volume which can compromise the final result of the restoration on the edentulous area, it is imperative to preserve the socket after extraction of a tooth.

\section{*Corresponding Author}

Dr. Khushbu Adhikari, Lecturer, Department of Periodontology and Oral Implantology, College of Dental Surgery, Universal College of Medical Sciences, Bhairahawa, Nepal.

E-mail: adhkhush@gmail.com
There are many techniques employed for preserving the width and height of ridge like socket seal using connective tissue graft, free gingival graft, alloderm, subepithelial connective tissue graft and guided bone regeneration(GBR) using membranes. The resorption process can be modified by adding hydroxyapatite, asynthetic bone graft which is primarily osteoconductive and provides a scaffold for the growth of cellular and vascular components to form new bone. It is structurally similar to bone and occupies only $10 \%$ of defect giving space of $90 \%$ for regeneration of new bone. ${ }^{3}$ Second generation autologous blood concentrates; Platelet Rich Fibrin(PRF)is a fibrin matrix which is rich in platelets and leukocytes. It consists of a variety of growth factors which is released in the site in a sustained manner for atleast 7 days. ${ }^{4}$ This case report describes a simple yet effective procedure of socket preservation by combining the alloplastic bone graft material and PRF to make a strong foundation for the placement of implant. 


\section{Case Report}

A 48 years old, male patient reported to the Department of Periodontics and Oral Implantology, UCMS College of Dental Surgery with the chief complaint of pain in the left posterior region of the upper jaw since 3 days. The pain was dull in nature which aggravated on chewing food and relieved after taking pain relieving medication. The medical history of the patient revealed that he was on medication for hypertension since 5 years. His past dental history revealed restoration of lower left posterior tooth 6 years back.

On clinical examination, tooth number 26 was grossly carious with Grade III mobility. Radiographic evaluation (Intraoral Periapical Radiograph) showed root stump of 26 with poor bone support (Fig.1). Extraction of tooth 26 was planned based on its gross carious involvement, periodontal status and radiographic findings. The replacement options for the resultant edentulous space were discussed with the patient in consultation with Department of Prosthodontics and Maxillofacial Prosthetics. The patient was duly informed about possible limitations and outcome of the procedure. After the patient gave consent, extraction of the root stump with socket preservation followed by implant placement was done.

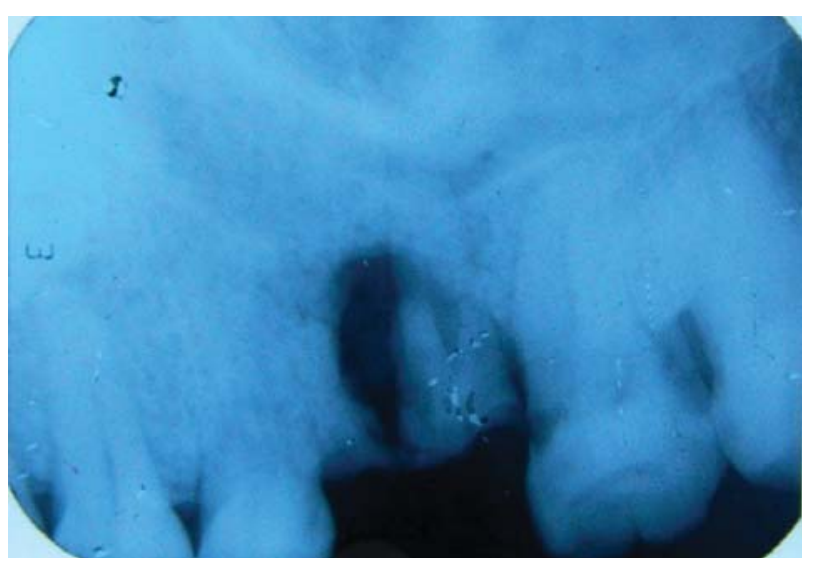

Figure 1: Pre-operative IOPAR
After formulating the treatment plan, a traumatic extraction of the root stump was done under local anesthesia. The socket walls were prepared using a surgical curette to debride it off of remnant granulation tissues. $10 \mathrm{ml}$ of venous blood from the patient was collected in a tube without an anticoagulant and immediately centrifuged at 3,000 revolutions per minute (rpm) for 10 minutes for preparing the PRF. The following three layers: upper straw-colored acellular plasma, red-colored lower fraction containing red blood cells (RBCs), and the middle fraction containing the fibrin clot were obtained. The socket was gently packed with a mixture of PRF and hydroxyapatite graft(150$700 \mu \mathrm{m})$ and covered by PRF membrane (Fig.2). To adapt the marginal soft tissues over the socket, cross-over sutures using 3-0 non-resorbable silk material was placed and a periodontal dressing was given. The patient was prescribed with antibiotics and analgesics. Oral hygiene and post-operative instructions were reinforced and the patient was followed-up after a week for suture removal and re-evaluation. Assessment of healing in the extraction socket was done at 3 and 6 months subsequently (Fig 3 and 4). Finally, an implant (TouaregTM $-S$, Adin Dental Implant Systems) of $5 \mathrm{~mm}$ diameter and $10 \mathrm{~mm}$ length was placed by indirect sinus lift technique (Fig.5).

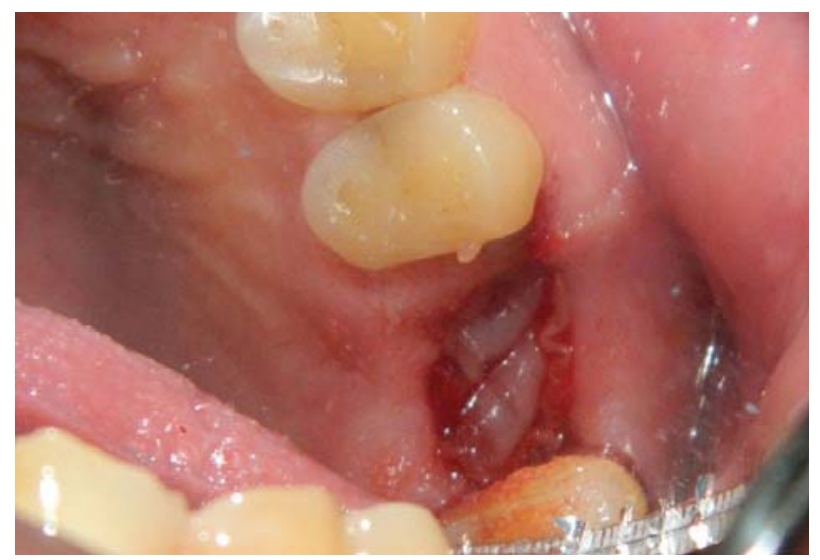

Figure 2: $P R F+H A$ covered with PRF membrane in the socket 


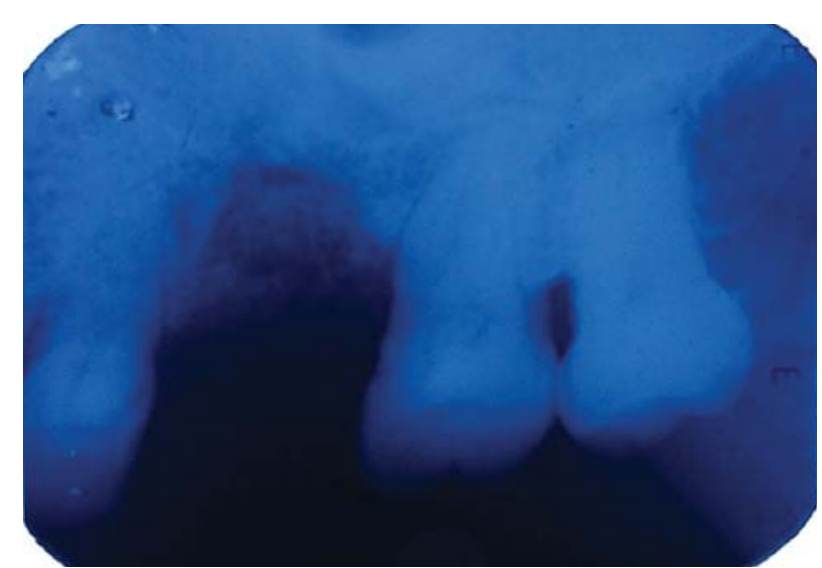

Figure 3: Post-operative IOPAR (3 months)

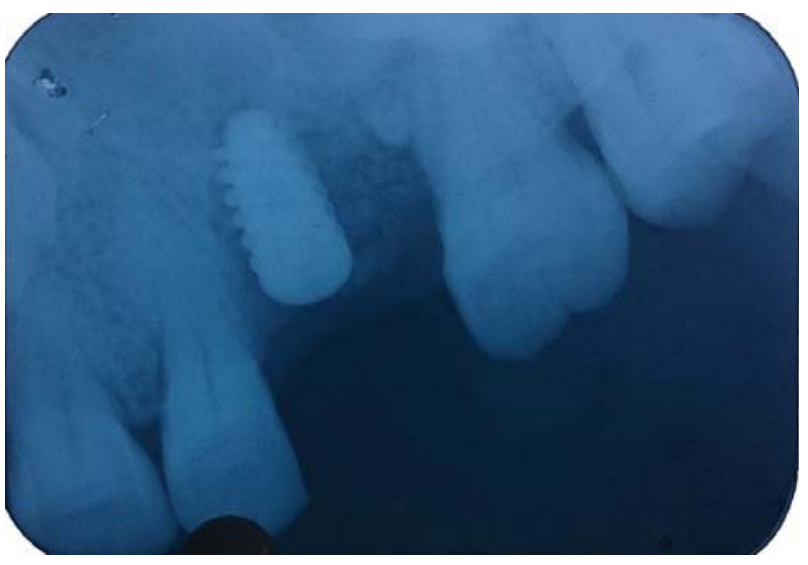

Figure 5: Implant placed

\section{Discussion}

The bone loss as a result of chronic periapical infection or severing of the blood vessels after any tooth extraction procedure is inevitable. The resorption process involves and affects the buccal plate more than the lingual plate. Various autogenous and alloplastic materials are commonly used to regenerate bone. Hydroxyapatite is a synthetic material exhibiting advantages like high abundance relative to natural materials, no risk of disease transmission and very low antigenicity which overcomes the inherent problems associated with autograft. ${ }^{5}$ The relative rough structure and large particle size, allows for adequate bone growth. ${ }^{6}$ The resorption rate of this material is slow, allowing it to act as a scaffold for bone replacement.

PRF plugs or membranes can also be used to fill extraction sockets, even when associated

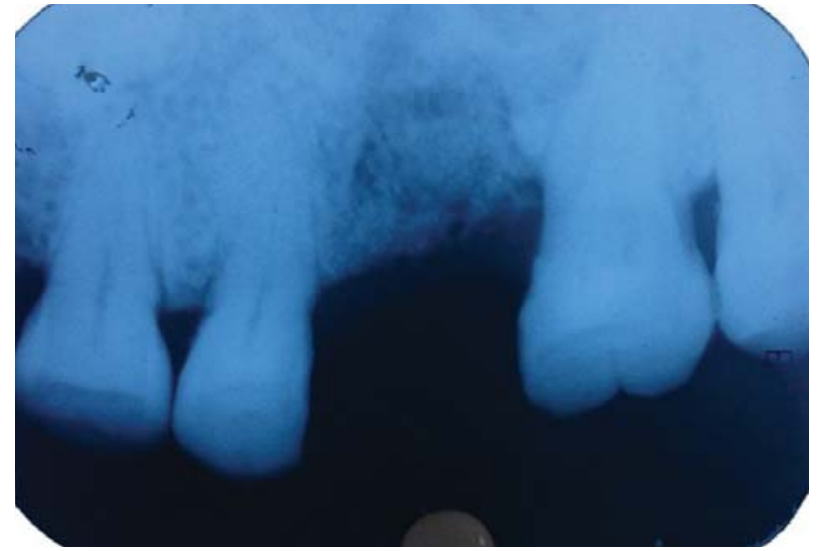

Figure 4: Post-operative IOPAR (6 months)

with compromised extraction sockets. ${ }^{7}$ The use of PRF with hydroxyapatite crystals not only supports the surgical site but also promotes soft tissue healing. PRF when mixed with graft material functions as a biological connector between the elements of graft particles and acts as a matrix which supports angiogenesis, capture of stem cells and migration of osteoprogenitor cells to the center of the graft. ${ }^{8}$ In addition, PRF membrane used as a protective cover over the grafted socket is particularly important when wound closure is impossible or difficult with the sutures alone. ${ }^{9}$

The advantages of use of PRF includes less surgical time, elimination of techniques and potential healing difficulties as compared to GBR procedures. ${ }^{11}$ Clinical and histological findings also suggested that filling PRF in a fresh extraction socket provides an alternative for implant site preparation by enhancing the natural coagulation process, stabilizing the clot and aids in socket healing. ${ }^{12}$ Hence, PRF with hydroxyapatite for this case was an effective method for laying a foundation of sound bone and tissue for implant placement.

\section{CONCLUSION}

Socket preservation technique using PRF and hydroxyapatite is an effective method to promote both soft and hard tissue healing and regeneration for implant placement. An increasing number of materials and methods 
are being used in individualized approaches to preserve the socket. Longitudinal studies regarding treatment protocols that are less invasive, more reproducible and less technique sensitive with constant revision of available biomaterials for socket preservation are much needed.

\section{References}

1. Mecall RA, Rosenfeld AL. Influence of residual ridge resorption patterns on implant fixture placement and tooth position 1. Int J Periodontics Restorative Dent 1991;11:8-23.

2. Seibert JS, Salama H. Alveolar ridge preservation and reconstruction. Periodontol 20001996 June, 11: 69-81

3. Kaushik BT, Jayakumar ND, Padmalatha O, Varghese S. Treatment of human intra bony periodontal defects with hydroxyapatite $+\beta$ tricalcium phosphate bone graft alone and in combination with platelet rich plasma: A randomized controlled trial. Indian J Dent Res 2011;22:505-10.

4. Dohan Ehrenfest Del Corso M, Diss A, Moujhi J, Charrier J B. Three- Dimensional architecture and cell composition of a Choukron's platelet rich fibrin clot and membrane. J Periodontol 2010 Apr;81 (4):546-555.

5. Shetty, V.; Han, T.J. Alloplastic materials in reconstructive periodontal surgery. Dent. Clin. North Am. 1991, 35, 521-530

6. Pallesen, L.; Schou, S.; Aaboe, M.; HjortingHansen, E.; Nattestad, A.; Melsen, F. Influence of particle size of autogenous bone grafts on the early stages of bone regeneration: A histologic and stereologic study in rabbit calvarium. Int. J. Oral Maxillofac. Implant. 2002, 17, 498-506.

7. Peck MT, Marnewick J, Stephen L. Alveolar ridge preservation using leukocyte and plateletrich fibrin: a report of a case. Case Rep Dent 2011: 345048.82.

8. Simonpieri A, Del Corso M, Sammartino G, Dohan Ehrenfest DM. The relevance of Choukroun's platelet-rich fibrin and metronidazole during complex maxillary rehabilitations using bone allograft. Part II: Implant surgery, prosthodontics, and survival. Implant Dent 2009;18:220-9.

9. Del Corso M, Vervelle A, Simonpieri A, Jimbo R, Inchingolo F, Sammartino G, DohanEhrenfest DM. Current knowledge and perspectives for the use of platelet-rich plasma (PRP) and platelet-rich fibrin (PRF) in oral and maxillofacial surgery part 1: Periodontal and dentoalveolar surgery. Curr Pharm Biotechnol 2012; 13: 1207-1230.

10. DelCorso, M.; Sammartino, G.; DohanEhrenfest, D.M. Clinical evaluation of a modified coronally advanced flap alone or in combination with a platelet-rich fibrin membrane for the treatment of adjacent multiple gingival recessions: a 6-month study". J. Periodontol., 2009, 80(11), 1694-1697.

11. Zhao JH, Tsai $\mathrm{CH}$, Chang YC. Clinical and histologic evaluations of healing in an extraction socket filled with platelet rich fibrin. J Dent Sci 2011; 6: 116-122. 